\title{
Simulation of Tsunami Effect by Seismic Wave Propagation in Hypoplastic Medium at Vicinity of Free Boundary
}

\author{
Anatoli Chigarev $^{1, *}$, Victor Polenov ${ }^{2}$, Pavel Shirvel $^{1}$ \\ ${ }^{1}$ Department Theoretical Mechanics, Belarusian National Technical University, Belarus \\ ${ }^{2}$ Department of Mathematics, Voronezh State University, Russia
}

Copyright $\bigcirc 2018$ by authors, all rights reserved. Authors agree that this article remains permanently open access under the terms of the Creative Commons Attribution License 4.0 International License

\begin{abstract}
A theoretical study of seismic waves propagation in a soil layer with a free surface has a great importance for a prediction in engineering decisions. Wave packets are radiated from an earthquake source and transfer energy. A transformation and a selection of wave packets occur in a process of wave propagating that why waves which arrive in a layer have a length considerably greater than a variation scale of heterogeneity in a medium in a layer near free surface. In the case, when the properties of different layers affect a relatively small degree on a behavior of the waves, an approximation of effective medium gives a fairly good solution. A model of a hypoplastic medium is used for a describing of some effects, which are observed in the time of seismic wave propagation. The model of hypoplastic medium allows describing many effects which are observed in granular soils. We consider a successive application of effective medium and ray methods in order to receive of approximate analytical solutions wishing to describe shear wave propagation in stratified layer, which lies on a half-space.
\end{abstract}

Keywords Wave Propagation, Hypoplastic Medium, Ray Method, Stratified Layer, Hypoplastic Model

\section{Introduction}

The model of elastic stratified medium widely is applied in seismology geotechnique [1-4]. In the case, when a medium constitutes from discrete layers, then it is necessary to solve the boundary problem for each layer. It is enough a laborious investigation [5]. The dynamic equations for inhomogeneous media are the differential equations with variable coefficients. As it is known for solving of these equations there are no general analytical methods. The most famous methods which are applied for solving of differential dynamical equations for inhomogeneous media are a ray method [6] and method of effective medium [7]. Some time ago a hypoplastic model was applied for an investigation of seismic wave propagation in a soil $[8,9]$. The model of hypoplastic medium allows describing many effects which are observed in granular soils. We consider a successive application of effective medium and ray methods in order to receive of approximate analytical solutions for description of shear wave propagation in stratified layer, which lies on a half - space.

As it is known a real soil is inhomogeneous, is usually stratified in a depth. We take the model of effective medium which has the same macroscopic properties as real inhomogeneous medium. The effective model can be received on a basic of experimental results in the form of phenomenological theory or on a basic of theoretical accounts. We take as effective model a hypoplastic medium $[8,9]$, which describes a medium with initial stresses increased in depth linearly. It is correct if a thickness of each layer is comparatively less than a thickness of a great layer.

For a solving of constitutive phenomenological equations, we apply a ray method. An application of this method is correct if a wave length (or a width of a wave packet) is less than a variation scale of effective properties. It is mean that macroscopic properties change monotonically in a depth.

\section{Formulation of Problem}

The layer of stratified granule medium lies on the surface $x_{1} \geq x_{10}$ (Fig. 1). A thickness of the layer is $L$. At $t=0$ the plane $x_{1}=x_{10}$ (the surface $S_{0}$ ) the wave begins to move with the velocity $\vartheta=\vartheta\left(x_{10}, t\right)$ in the directions $x_{2}$. Then there is the plane shear wave which 
propagates in the direction $x_{1}$. At first we consider a propagation of energy in any layer.

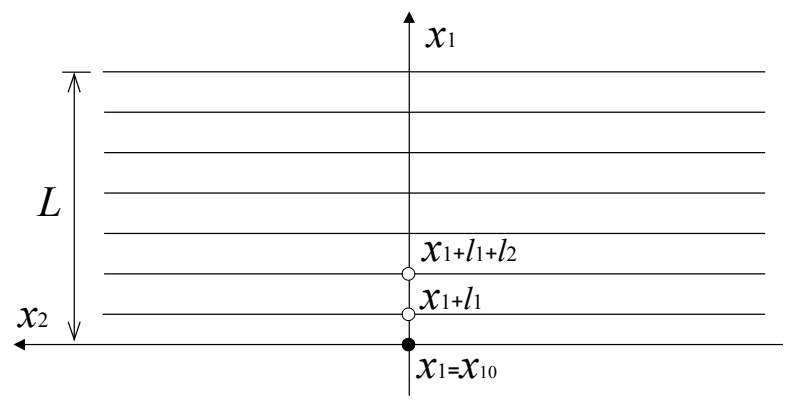

A propagation of wave packet in continuum is described of the first law of thermodynamic [10]

$$
\dot{K}+\dot{U}=Q+W,
$$

where $K=\frac{1}{2} \int_{V} \bar{\vartheta} \cdot \bar{\vartheta} \rho d V$ is a kinetic energy, $U=\int_{V} u \rho d V$ is an internal energy, $u(x, t)$ is a specific internal energy of unit mass, $W=\int_{V} \bar{F} \cdot \bar{Q} \rho d V+\int_{S} \operatorname{tr}\left\{\overline{t_{n}} \cdot \bar{v}\right\} d S$ is a power of generalized mass forces $F_{k}$ and surface forces $\bar{t}, Q$ is heat power.

Figure 1. The stratified layer $L$ which lies on half-space $x<x_{10}$

For constant density and isothermical processes

$$
\frac{d}{d t}(K+U)=\int_{V} \bar{F} \cdot \bar{\vartheta} \rho d V+\int_{S} \operatorname{tr}\left\{\bar{t}_{n} \cdot \bar{\vartheta}\right\} d S=\int_{V} F_{i} \vartheta_{i} \rho d V+\int_{S} t_{i j} \vartheta_{i} n_{j} d S,
$$

where $t_{i j}$ is stress tensor.

Let $V$ be a volume of current pipe, $S$ is a surface of current pipe (Fig. 2) then we obtain

$$
\frac{d}{d t}(K+U)=\int_{V} F_{i} \vartheta_{i} \rho d V+\int_{S_{1}} t_{i j} \vartheta_{i} n_{j} d S-\int_{S_{2}} t_{i j} \vartheta_{i} n_{j} d S, \quad S=S_{1}+S_{2} .
$$

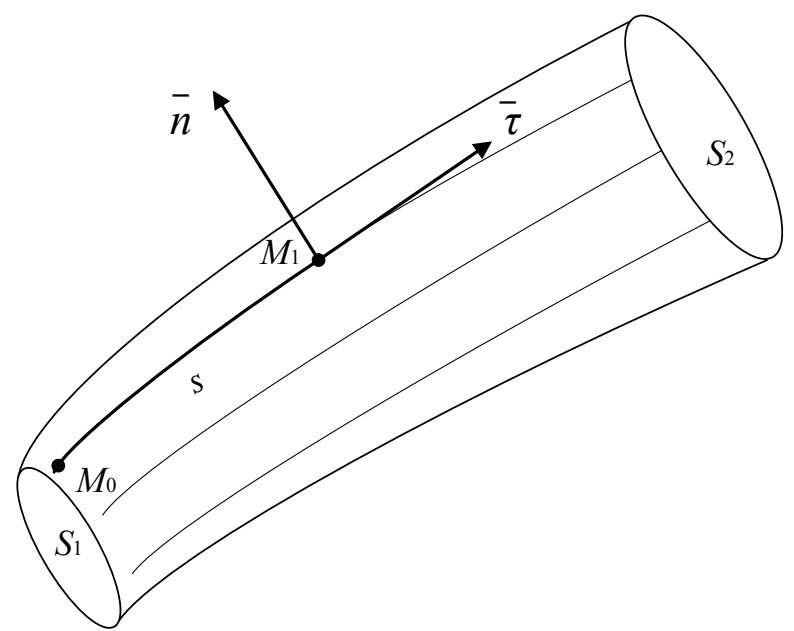

Figure 2. The ray pipe, which is formed by rays

Used a theorem about an average to the integrals in Eq. 1, we obtain [11]

$$
\frac{d}{d t}(K+U)=\bar{F} \cdot d \bar{r}+\left(t_{i j} \vartheta_{i}\right),{ }_{j} d t-t_{i j} \nabla_{j} \vartheta_{i} d t,
$$

where first member $\bar{F} \cdot d \bar{r}$ in right part is a work density of mass forces, second member $\left(t_{i j} \vartheta_{i}\right),{ }_{j} d t$ is a work density of surface forces, third member $-t_{i j} \nabla_{j} \vartheta_{i} d t$ is density of internal surface forces.

We have used the Eq. 3 in pure mechanical formulation (without a registration of a mechanic energy transformation to thermal energy and either kinds of energy). 
The Umov's - Poyinting's vector $\bar{P}$ (a density of flow energy) describes a direction of energy transmission in a medium.

Lines of energy flow are found from the equation [12]

$$
\frac{d \bar{r}}{d s}=\bar{\tau}=\frac{\bar{P}}{|\bar{P}|}, \quad P_{j}=t_{i j} \vartheta_{i}, \quad i, j=1,2
$$

where $\bar{r}(x, y, z)$ is radius vector of a point on an energy flow line, $S$ is a distance along a flow line, $\bar{\tau}$ is unit tangential of the vector to a flow line, $t_{i j}$ is stress tensor, $\vartheta_{i}$ is a velocity vector.

A variation of the vector $\bar{\tau}$ along a flow line is described of the formula

$$
\frac{d \bar{\tau}}{d s}=k \bar{n}, \quad k=1 / R,
$$

where $\bar{n}$ is normal vector, $k$ is curvature of a flow line, $R$ is curvature radius of a flow line.

A flow line curvature depends on medium parameters. We must set boundary conditions for each layer.

\section{Shear Wave Propagation in Stratified Granular Layer with Effective Hypoplastic Properties}

Let a layer of a thickness $L$ be on a half-space $x_{1} \leq x_{10}$. The layer $L$ constitutive from some layers of thicknesses $l_{i}(i=1,2, \ldots, n)$, it being know that $l_{i}<<L$, $(i=1,2, \ldots, n)$. For example, it may be an interchange of layers of sand and clay (Fig. 1).

Let a shear wave $(\mathrm{SH})$ be to incidences on a plane boundary $x_{1}=x_{10}$ and is propagated from the half-space $x_{1}<x_{10}$. If we want to solve the problem exactly we must set boundary conditions (or connected conditions) and describe wave propagation for each layer. There are very unwieldy expressions especially for multiple scattered waves.

A method of effective medium allows obtaining a solution of this problem [7]. Applied an averaging method (method of homogenization or energy continuation) we obtain a phenomenological model of inhomogeneous medium [7].

Suppose that macroscopic (effective) conditions of layer medium are described of the equations of a hypoplastic medium $[8,9]$.

In general case the equations of wave propagation in hypoplastic medium have the form $[6,7]$

$$
\operatorname{Div} T+\rho^{*} \bar{f}=\frac{d \rho \bar{\vartheta}}{d t}
$$

where $\bar{f}$ is a mass force vector, $\rho \bar{\vartheta}$ is a vector of quantity of motion, $\bar{\vartheta}$ is a rate vector, $\rho^{*}=\frac{\rho_{l} e+\rho_{s}}{1+e}$ is effective (average) density, $\rho_{l}$ and $\rho_{s}$ are densities of liquid and hard fraction in a soil respectively, $T$ is a tensor of effective (total) stresses in an inhomogeneous medium, $e$ is a pore quantity.

The kinetic equations we write in the form of constitutive equations of a hypo plasticity $[8,9]$

$$
\stackrel{\circ}{T}=H(T, D, e),
$$

where $\stackrel{\circ}{T}$ is Jaunman's derivative time.

$$
\stackrel{\circ}{T}=\dot{T}-T \omega-\omega T,
$$

in (8) $\dot{T}$ is material derivative time, $D$ and $\omega$ are a tensor of rate and a spin tensor respectively.

$$
D_{i j}=\frac{1}{2}\left(\frac{\partial \vartheta_{i}}{\partial x_{j}}+\frac{\partial \vartheta_{j}}{\partial x_{i}}\right), \quad \omega_{i j}=\frac{1}{2}\left(\frac{\partial \vartheta_{i}}{\partial x_{j}}-\frac{\partial \vartheta_{j}}{\partial x_{i}}\right) \text {. (९) }
$$

A density $\rho^{*}$ satisfies the equation of continuity

$$
\frac{d \rho^{*}}{d t}+\operatorname{div}\left(\rho^{*} \bar{\vartheta}\right)=0
$$

and pore quantity $e$ satisfies the equation

$$
\dot{e}=(1+e) \operatorname{tr} D \text {. }
$$

We represent the each field value $T_{i j}(\bar{x}, t), \vartheta_{i}\left(\bar{x}_{i}, t\right)$, $\bar{u}_{i}(\bar{x}, t), \rho(\bar{x}, t), e(\bar{x}, t)$ which describes a dynamical state in hypoplastic medium in the form of the sum

$$
\begin{gathered}
T=T^{\circ}+\widetilde{T}, \bar{\vartheta}=\bar{\vartheta}^{\circ}+\widetilde{\vartheta}, \bar{u}=\bar{u}^{\circ}+\widetilde{u}, \\
\rho^{*}=\rho^{\circ}+\widetilde{\rho}, e^{*}=e^{\circ}+\widetilde{e},
\end{gathered}
$$

where $T^{\circ}, \bar{\vartheta}^{\circ}, \bar{u}^{\circ}, \rho^{\circ}, e^{\circ}$ describe initial state and $\widetilde{T}, \widetilde{\vartheta}, \tilde{u}, \tilde{\rho}, \tilde{e}$ describe disturbances.

The initial stresses $T^{\circ}(x, t)$ satisfies to the equations of equilibrium

$$
\operatorname{Div} T^{\circ}-\operatorname{grad} \bar{P}_{l}^{\circ}+\rho \bar{f}=0,
$$

then the equations for disturbances have the form

$$
\operatorname{Div} T-\operatorname{grad} P_{l}=\frac{d \rho \vartheta}{d t},
$$

Here and in the future the sign $\sim$ we do not write. Let Eq. (7) be the form

$$
\stackrel{\circ}{T}=L(T, e) D+N(T, e)\|D\|
$$


The expression (14) is written in nonindex form (directly designation). In the index form the members of equation (14) have form

$$
\begin{aligned}
& L_{i j k l}=\frac{f_{b} f_{e}}{\operatorname{tr}\left(\widehat{T}^{2}\right)}\left[F^{2} \delta_{i k} \delta_{j l}+a^{2} \widehat{T}_{i j} \widehat{T}_{k l}\right], L_{i j}=\frac{f_{b} f_{e} f_{\alpha} a F}{\operatorname{tr}\left(\widehat{T}^{2}\right)}\left[\widehat{T}_{i j}+\widehat{T}_{i j}^{*}\right], \\
& \widehat{T}_{i j}=\frac{T_{i j}}{t r T}, \widehat{T}_{i j}^{*}=\widehat{T}_{i j}-\frac{1}{3} \delta_{i j}, a=\sqrt{\frac{3}{8}} \frac{3-\sin \varphi_{c}}{\sin \varphi_{c}},
\end{aligned}
$$

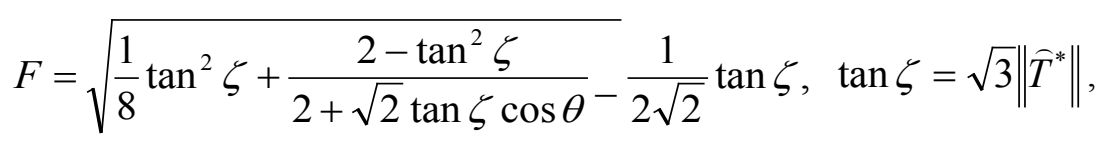

$$
\begin{aligned}
& \cos 3 \theta=-\sqrt{6} \frac{\operatorname{tr}\left(\widehat{T}^{* 3}\right)}{\left[\operatorname{tr}\left(\widehat{T}^{* 2}\right)\right]^{3 / 2}}, f_{\alpha}=\left(\frac{e-e_{\alpha}}{e_{c}-e_{\alpha}}\right), f_{\alpha}=\left(\frac{e_{c}}{e}\right)^{\beta}, \\
& f_{b}=\frac{h_{s}}{n}\left(\frac{1+e_{i}}{e_{c}}\right)\left(\frac{e_{i^{\circ}}}{e_{c^{\circ}}}\right)^{\beta}\left(\frac{-t r T}{h_{s}}\right)^{1-n}\left[3+a^{2}-\sqrt{3 a}\left(\frac{e_{i^{\circ}}-e_{\alpha^{\circ}}}{e_{c^{\circ}}-e_{\alpha^{\circ}}}\right)\right]^{-1}, \\
& \frac{e_{i}}{e_{i^{\circ}}}=\frac{e_{c}}{e_{c^{\circ}}}=\frac{e_{\alpha}}{e_{\alpha^{\circ}}}=\exp \left[-\left(\frac{-t r T}{h_{s}}\right)^{n}\right], e_{i^{\circ}}>e_{c^{\circ}}>e_{\alpha^{\circ}}
\end{aligned}
$$

where $e_{\alpha}$ is the minimal possible void ratio, $e_{c}$ is the critical void ratio, $e_{i}$ is void ratio in the least state, $L, \beta, n, h$ are material parameters, $\varphi_{c}$ is the friction angle in critical state. For example, in Table 1 the constitutive hypoplastic parameters of Hochstetten sand are given $[8,9]$.

Table 1. Hypoplastic parameters of Hochstetten sand $[8,9]$

\begin{tabular}{|c|c|c|c|c|c|c|c|}
\hline$\varphi_{c}\left[^{\circ}\right]$ & $h_{s}[\mathrm{MPa}]$ & $e_{\alpha^{\circ}}$ & $e_{c^{\circ}}$ & $e_{i^{\circ}}$ & $L$ & $\beta$ & $h$ \\
\hline 33 & 1000 & 0,95 & 0,55 & 1,05 & 0,25 & 1,5 & 0,25 \\
\hline
\end{tabular}

The equation for disturbances follows from Eq. 9 for case when $\widehat{T}_{i j}^{*}$ is not enough

$$
\dot{T}=L\left(T^{\circ}, e\right) D+N\left(T^{\circ}, e\right)\|D\| .
$$

Therefore the Eqs. 5, 6, 8, 10 describe a disturbance propagation in hypoplastic medium.

\section{Shear Wave Propagation in Effective Hypoplastic Medium}

Set initial and boundary conditions on plane $x_{1}=x_{10}$ in the form

$$
\begin{aligned}
& \left.\vartheta_{2}\left(x_{1}, t\right)\right|_{t=0}=\vartheta_{2}\left(x_{1}, 0\right),\left.\quad \vartheta_{2}\left(x_{1}, t\right)\right|_{x_{1}=x_{10}}=\vartheta_{2}\left(x_{10}, t\right), \\
& \left.T_{12}\left(x_{1}, t\right)\right|_{t=0}=T_{12}\left(x_{1}, 0\right),\left.\quad T_{12}\left(x_{1}, t\right)\right|_{x_{1}=x_{10}}=T_{12}\left(x_{10}, t\right),
\end{aligned}
$$

The motion equation Eq. 8 in this case has the form

$$
\begin{aligned}
& \frac{\partial T_{11}}{\partial x_{1}}-\frac{\partial P_{l}}{\partial x_{1}}=0, \\
& \frac{\partial T_{12}}{\partial x_{1}}-\rho \frac{\partial \vartheta_{2}}{\partial x_{1}}=0,
\end{aligned}
$$


The coefficients $K_{i}$ we write analogous $[6,7]$ in the form

Suppose that for an initial state it has place the condition $T_{12}^{\circ}\left(x_{1}\right)=0$ we obtain:

$$
\begin{array}{ll}
\frac{\partial T_{11}}{\partial x_{1}}-K_{2}\left|\frac{\partial \vartheta_{2}}{\partial x_{1}}\right|=0, & \frac{\partial T_{12}}{\partial t}-K_{3} \frac{\partial \vartheta_{2}}{\partial x_{1}}=0, \\
\frac{\partial T_{22}}{\partial t}-K_{6}\left|\frac{\partial \vartheta_{2}}{\partial x_{1}}\right|=0, & \frac{\partial T_{33}}{\partial t}-K_{8}\left|\frac{\partial \vartheta_{2}}{\partial x_{1}}\right|=0 .
\end{array}
$$

Combined Eq. 24, Eq. 25, Eq. 21 we obtain the equations for wave disturbance propagation in the layer with effective properties

$$
\begin{array}{r}
\frac{\partial}{\partial t}\left(\rho\left(x_{1}\right) \frac{\partial \vartheta_{2}}{\partial t}\right)-\frac{\partial}{\partial x_{1}}\left(K_{3}\left(x_{1}\right) \frac{\partial \vartheta_{2}}{\partial x_{1}}\right)=0 \\
\frac{1}{K_{3}\left(x_{1}\right)} \frac{\partial^{2} T_{12}}{\partial t^{2}}=\frac{\partial}{\partial x_{1}}\left(\frac{1}{\rho\left(x_{1}\right)} \frac{\partial T_{12}}{\partial x_{1}}\right)=0 .
\end{array}
$$

where

$$
\begin{array}{ll}
K_{1}=-T_{12}^{\circ}+h_{2} \widehat{T}_{12}^{\circ} \widehat{T}_{11}^{\circ}, & K_{2}=\sqrt{2} h_{3}\left(\widehat{T}_{11}^{\circ}-1 / 6\right), \\
K_{3}=1 / 3\left[T_{11}^{\circ}-T_{22}^{\circ}\right]+h_{1} / 2+h_{2}\left(\widehat{T}_{12}^{\circ}\right)^{2}, & K_{4}=\sqrt{2} h_{3} \widehat{T}_{12}^{\circ}, \\
K_{5}=T_{12}^{\circ}+h_{2} \widehat{T}_{12}^{\circ} \widehat{T}_{22}^{\circ}, & K_{6}=\sqrt{2} h_{3}\left(\widehat{T}_{22}^{\circ}-1 / 6\right), \\
K_{7}=h_{2} \widehat{T}_{12}^{\circ} \widehat{T}_{33}^{\circ}, & K_{8}=\sqrt{2} h_{3}\left(\widehat{T}_{33}^{\circ}-1 / 6\right),
\end{array}
$$

\section{Solving of Equations of Shear Wave Propagation in Layer}

The differential equations Eq. 26, Eq. 27 have variable coefficients. There are no general analytical methods for a solving of similar equations. The ray method is the most effective among different asymptotic methods for solving of differential equations with variable coefficients [6].

For nonstationary waves an application of this method is correct, if a wave length $\lambda$ is much less than a variable of a scale of effective layer parameters. It has place if an inhomogeneity of a layer changes in a depth monotonic.

It is known that usually a stiffness of a layer changes local nonmonotonic in a depth, but effective stiffness is monotonic function of spatial coordinate in a depth.

Let the coefficients $K_{i}\left(x_{1}\right), \rho\left(x_{1}\right)$ in the equations Eq. 26, Eq. 27 be are effective parameters of medium and a scale of variable $K_{i}, \rho$ is more greater than a wave length. An effective approximation gives us principal estimation of wave field values caused of integral (average) conditions of real medium.

Write the solution of the equations Eq. 26, Eq. 27 in the form

$$
\begin{gathered}
\vartheta_{2}\left(x_{1}, t\right)=\sum_{n=o}^{\infty} \vartheta_{2}^{(n)}\left(x_{1}\right) f_{n}\left(t-\psi\left(x_{1}\right)\right), \\
T_{12}\left(x_{1}, t\right)=\sum_{n=o}^{\infty} T_{12}^{(n)}\left(x_{1}\right) f_{n}\left(t-\psi\left(x_{1}\right)\right), \\
\frac{\partial f_{n}(\xi)}{\partial \xi}=f_{n-1}(\xi),
\end{gathered}
$$

where $\psi\left(x_{1}\right)$ is eiconal, $\vartheta_{2}^{(n)}\left(x_{1}\right), T_{12}^{(n)}\left(x_{1}\right)$ are unknown quantities. 
If $f_{n}\left(t-\psi\left(x_{1}\right)\right)=\frac{\left[t-\psi\left(x_{1}\right)\right]^{n}}{n !}$ then ray series Eq. 29, Eq. 30 have form

$$
\begin{aligned}
& \vartheta_{2}^{(n)}\left(x_{1}, t\right)=\sum_{n=0}^{\infty} \vartheta_{2}^{(n)}\left(x_{1}\right) \frac{\left[t-\psi\left(x_{1}\right)\right]^{n}}{n !}, \\
& T_{12}^{(n)}\left(x_{1}, t\right)=\sum_{n=0}^{\infty} T_{12}^{(n)}\left(x_{1}\right) \frac{\left[t-\psi\left(x_{1}\right)\right]^{n}}{n !} .
\end{aligned}
$$

If $f_{0}$ is Heaviside function $H(\tau)$ then $f_{n}$ has form

$$
f_{n}=\frac{\left[t-\psi\left(x_{1}\right)\right]^{n}}{n !} H\left(t-\psi\left(x_{1}\right)\right) .
$$

Substituted Eq. 32, Eq. 33 into Eq. 26, Eq. 27 we obtain

$$
\begin{aligned}
& \sum_{n=o}^{\infty}\left\{\left[\left(\psi^{\prime}\right)^{2} K_{3}-\rho\right] \vartheta_{2}^{(n)} f_{n-2}-\left[2 K_{3} \psi^{\prime} \vartheta_{2}^{(n)}+\left(K_{3}^{\prime} \psi^{\prime}\right) \vartheta_{2}^{(n)}\right] f_{n-1}+\left[K_{3} \vartheta_{2}^{(n)^{\prime \prime}}+K_{3} \vartheta_{2}^{(n)}\right] f_{n}\right\}=0 \\
& \sum_{n=o}^{\infty}\left\{\left[\left(\psi^{\prime}\right)^{2} K_{3}-\rho\right] T_{12}^{(n)} f_{n-2}-\left[2 K_{3} \psi^{\prime} T_{12}^{(n)^{\prime}}+\left(K_{3}^{\prime} \psi^{\prime}\right) T_{12}^{(n)}\right] f_{n-1}+\left[K_{3} T_{12}^{(n)}{ }^{\prime \prime}+K_{3} T_{12}^{(n)}\right] f_{n}\right\}=0 .
\end{aligned}
$$

For $n<0$ it must be $\vartheta_{2}^{(n)}=0, T_{12}^{(n)}=0$ because the coefficient at $f_{0}$ is equal 0 .

From Eq. 35, Eq. 36 it follows

$$
\begin{gathered}
{\left[\left(\psi^{\prime}\right)^{2} K_{3}-\rho\right] \vartheta_{2}^{(n+1)}-2 K_{3} \psi^{\prime} \vartheta_{2}^{(n)}-\left(K_{3} \psi^{\prime \prime}+K_{3} \psi^{\prime}\right) \vartheta_{2}^{(n)}+K_{3} \vartheta_{2}^{(n-1)^{\prime \prime}}+K_{3}^{\prime} \vartheta_{2}^{(n-1)}{ }^{\prime}=0,} \\
{\left[\left(\psi^{\prime}\right)^{2} K_{3}-\rho\right] T_{12}^{(n+1)}-2 K_{3} \psi^{\prime} T_{12}^{\prime(n)}-\left(K_{3} \psi^{\prime \prime}+K_{3} \psi^{\prime}\right) T_{12}^{(n)}+K_{3} T_{12}^{\prime \prime(n-1)}+K_{3}^{\prime} T_{12}^{(n-1)}=0 .}
\end{gathered}
$$

For $n=-1$ we obtain $\vartheta_{2}^{(n)}=0, T_{12}^{(n)}=0$ but $\vartheta_{2}^{(0)} \neq 0, T_{12}^{(0)} \neq 0$ and therefore from Eq. 37, Eq. 38 it follows

$$
\left(\psi^{\prime}\left(x_{1}\right)\right)^{2}=\frac{\rho\left(x_{1}\right)}{K_{3}\left(x_{1}\right)}=C_{S}^{-2}\left(x_{1}\right),
$$

where $C_{S}^{-2}\left(x_{1}\right)$ is a velocity of shear wave.

The solution of Eq. 39 is written in the form

$$
\psi\left(x_{1}\right)=\psi\left(x_{10}\right) \pm \int_{x_{10}}^{x_{1}} \frac{d x_{1}}{C_{S}\left(x_{1}\right)}
$$

In the formula Eq. 40 we take the sign "+" for waves which propagate in the direction $+x_{1}$ and the sign "--" for waves in direction $-x_{1}$.

Substituted Eq. 40 into Eq. 37, Eq. 38 we obtain the equations of transfer for $\vartheta_{2}^{(n)}\left(x_{1}\right), T_{12}^{(n)}\left(x_{1}\right)$. The equations Eq. 37, Eq. 38 with a registration Eq. 40 are solved in general form

$$
\begin{gathered}
\vartheta_{2}^{(n)}\left(x_{1}\right)=\vartheta_{2}^{(n)}\left(x_{10}\right)\left[\frac{\rho\left(x_{10}\right) K_{3}\left(x_{10}\right)}{\rho\left(x_{1}\right) K_{3}\left(x_{1}\right)}\right]^{1 / 4}+\int_{x_{10}}^{x_{1}} \vartheta_{2}^{(n-1)}(\tau)\left[\frac{\rho(\tau) K_{3}(\tau)}{\rho\left(x_{1}\right) K_{3}\left(x_{1}\right)}\right]^{1 / 4} d \tau \\
\vartheta_{2}^{(n-1)}=\frac{ \pm 1}{2\left(\rho K_{3}\right)^{1 / 2}} \cdot \frac{d}{d x_{1}}\left[K_{3} \vartheta_{2}^{(n-1)^{\prime}}\right],
\end{gathered}
$$




$$
\begin{gathered}
T_{12}^{(n)}\left(x_{1}\right)=T_{12}^{(n)}\left(x_{10}\right)\left[\frac{\rho\left(x_{10}\right) K_{3}\left(x_{10}\right)}{\rho\left(x_{1}\right) K_{3}\left(x_{1}\right)}\right]^{1 / 4}+\int_{x_{10}}^{x_{1}} P_{12}^{(n-1)}(\tau)\left[\frac{\rho\left(x_{1}\right) K_{3}\left(x_{1}\right)}{\rho(\tau) K_{3}(\tau)}\right]^{1 / 4} d \tau, \\
P_{12}^{(n-1)}= \pm \frac{\left(\rho K_{3}\right)^{1 / 2}}{2} \cdot \frac{d}{d x_{1}}\left[\frac{T_{12}^{(n-1)^{\prime}}}{\rho}\right] .
\end{gathered}
$$

In formulas Eq. 41, Eq. 43 the members at $n=0$ are principal because they describe main part of wave energy. Supposed $n=-1,0$ in the equations Eq. 37, Eq. 38 we obtain the equations Eq. 39, Eq. 40 and the equations of transfer

$$
\begin{aligned}
& +2 K_{3} \psi^{\prime} \vartheta_{2}^{(0)}+\left(K_{3} \psi^{\prime \prime}+K_{3} \psi^{\prime}\right) \vartheta_{2}^{(0)}=0 \\
& +2 K_{3} \psi^{\prime} T_{12}^{(0)}+\left(K_{3} \psi^{\prime \prime}+K_{3} \psi^{\prime}\right) T_{12}^{(0)}=0 .
\end{aligned}
$$

Multiplied Eq. 45 on $V_{2}^{(0)}$ and Eq.46 on $T_{12}^{(0)}$ we obtain

$$
\begin{aligned}
& K_{3} \psi^{\prime}\left(\vartheta_{2}^{(0)^{2}}\right)^{\prime}+\left(K_{3} \psi^{\prime}\right) \vartheta_{2}^{(0)^{2}}=0 \\
& K_{3} \psi^{\prime}\left(T_{12}^{(0)^{2}}\right)^{\prime}+\left(K_{3} \psi^{\prime}\right) T_{12}^{(0)^{2}}=0
\end{aligned}
$$

or transformed we obtain the law of conversation of energy along ray pipes

$$
\begin{aligned}
& \left(K_{3} \psi^{\prime} \vartheta_{2}^{(0)^{2}}\right)^{\prime}=0, \quad P_{2}^{(0)}=K_{3} \psi^{\prime} \vartheta_{2}^{(0)^{2}} ; \\
& \left(K_{3} \psi^{\prime} \vartheta_{2}^{(0)^{2}}\right)^{\prime}=0, \quad P_{12}^{(0)}=K_{3} \psi^{\prime} T_{12}^{(0)^{2}}
\end{aligned}
$$

Chose a ray pipe in the form of a cylinder with area of a base $d S_{0}$ and with an altitude $L$ Fig. 3 .

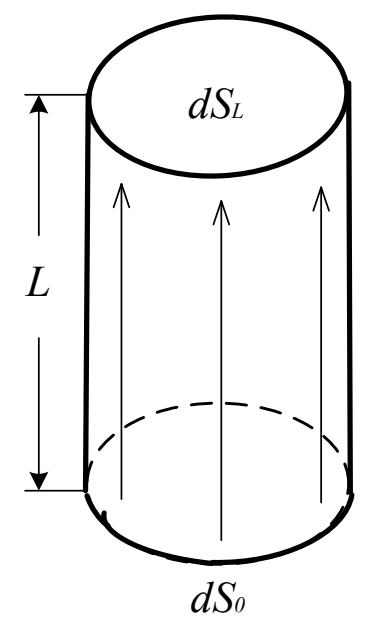

Figure 3. The ray pipe in the layer $L$, which is perpendicularity to free surface of half-space

The expressions 47, 48 in 3-D case are written in the form

$$
\begin{aligned}
& \nabla \cdot \bar{P}_{2}^{(0)}=0, \quad \bar{P}_{2}^{(0)}=\left|u_{2}^{(0)}\right|^{2} \bar{k}_{\vartheta}, \\
& \nabla \cdot \bar{P}_{12}^{(0)}=0, \quad \bar{P}_{12}^{(0)}=\left|T_{12}^{(0)}\right|^{2} \bar{k}_{\sigma},
\end{aligned}
$$


where $\bar{k}_{\vartheta}=\operatorname{grad} \psi_{v}, \bar{k}_{\sigma}=\operatorname{grad} \psi_{\sigma}, \bar{P}_{2}^{(0)}$ and $\bar{P}_{12}^{(0)}$ are the vectors of flow density of energy.

Integrated Eq. 49, Eq. 50 on a volume of ray pipe and applied Gauss theorem we obtain

$$
\begin{aligned}
& \oint_{S} \bar{P}_{2}^{(0)} \cdot \bar{n} d s=0, \\
& \oint_{S} \bar{P}_{12}^{(0)} \cdot \bar{n} d s=0,
\end{aligned}
$$

where $S$ is closed surface of ray pipe, $\bar{n}$ is normal vector to $S$ [17]

From Eq. 51 follow the conditions

$$
\begin{aligned}
& P_{2}^{(0)}(L)=P_{2}^{(0)}(0) \frac{d S_{0}}{d S_{L}}, \\
& P_{12}^{(0)}(L)=P_{12}^{(0)}(0) \frac{d S_{0}}{d S_{L}},
\end{aligned}
$$

where $d S$ is the area of a base of ray pipe at $x_{1}=x_{10}$ and $d S_{L}$ is an area of upper base of ray pipe at $x_{1}=x_{10}+L$.

As $d S_{(0)}=d S_{L}$ so obtain the law conversation of low density of energy

$$
P_{2}^{(0)}(L)=P_{2}^{(0)}(0), \quad P_{12}^{(0)}(L)=P_{12}^{(0)}(0) .
$$

The solutions of the Eqs. 45, 46 have the form

$$
\begin{aligned}
& \vartheta_{2}^{(0)}\left(x_{1}\right)=\vartheta_{2}^{(0)}\left(x_{10}\right) \sqrt[4]{\frac{K_{3}\left(x_{10}\right) \rho\left(x_{10}\right)}{K_{3}\left(x_{1}\right) \rho\left(x_{1}\right)}}, \\
& T_{12}^{(0)}\left(x_{1}\right)=T_{12}^{(0)}\left(x_{10}\right) \sqrt[4]{\frac{K_{3}\left(x_{1}\right) \rho\left(x_{1}\right)}{K_{3}\left(x_{10}\right) \rho\left(x_{10}\right)}} .
\end{aligned}
$$

Let initial impulse be an impulse function $f_{0}(t)$ in the form

$$
f_{0}(t)=J_{0}(t, \tau)= \begin{cases}0, & t<0 \\ 1, & 0 \leq t \leq \tau, \\ 0, & \tau<t\end{cases}
$$

where the function $J_{0}(t, \tau)$ is expressed with the help-of Heaviside function

$$
J_{0}(t, \tau)=H_{0}(t)-H_{0}(t-\tau)
$$

The boundary conditions by $x_{1}=x_{10}$ have the form

$$
\begin{aligned}
& \vartheta_{2}^{(0)}\left(x_{10}, t\right)=\vartheta_{2}^{(0)}\left(x_{10}\right) J_{0}(t, \tau) \\
& T_{12}^{(0)}\left(x_{10}, t\right)=T_{12}^{(0)}\left(x_{10}\right) J_{0}(t, \tau)
\end{aligned}
$$

The wave which comes in the point $x_{1}$ is described with a registration Eq. 55 in the formulas

$$
\begin{aligned}
& \vartheta_{2}^{(0)}\left(x_{1}, t\right)=\vartheta_{2}^{(0)}\left(x_{10}\right) \sqrt[4]{\frac{K_{3}\left(x_{10}\right) \rho\left(x_{10}\right)}{K_{3}\left(x_{1}\right) \rho\left(x_{1}\right)}} J_{0}\left(t-\tau-\int_{x_{10}}^{x_{1}} \frac{d x_{1}}{C_{S}\left(x_{1}\right)}\right) \\
& T_{12}^{(0)}\left(x_{1}, t\right)=T_{12}^{(0)}\left(x_{10}\right) \sqrt[4]{\frac{K_{3}\left(x_{10}\right) \rho\left(x_{1}\right)}{K_{3}\left(x_{10}\right) \rho\left(x_{10}\right)}} J_{0}\left(t-\tau-\int_{x_{10}}^{x_{1}} \frac{d x_{1}}{C_{S}\left(x_{1}\right)}\right)
\end{aligned}
$$

The conditions Eq. 54 allow to obtain a variation of wave profile. With a registration Eq. 58 , Eq. 59 we have

$$
\begin{aligned}
& J_{0}\left(t_{1}, \tau\right)=\sqrt[4]{\frac{K_{3}\left(x_{0}\right) \rho\left(x_{0}\right)}{K_{3}(x) \rho(x)}} J_{0}\left(t-\tau-\int_{x_{10}}^{x_{1}} \frac{d x_{1}}{C_{S}\left(x_{1}\right)}\right) \text { for } \vartheta_{2}^{(0)} \\
& J_{0}\left(t_{1}, \tau\right)=\sqrt[4]{\frac{K_{3}(x) \rho(x)}{K_{3}\left(x_{0}\right) \rho\left(x_{0}\right)}} J_{0}\left(t-\tau-\int_{x_{10}}^{x_{1}} \frac{d x_{1}}{C_{S}\left(x_{1}\right)}\right) \text { for } T_{12}^{(0)}
\end{aligned}
$$

Integrated on $t$ the left part of Eq. 60 from $O$ up to $\tau\left(x_{1}\right)$ we obtain

$$
\begin{aligned}
\tau_{\vartheta} & =\tau_{1 \vartheta} \sqrt[4]{\frac{K_{3}\left(x_{1}\right) \rho\left(x_{1}\right)}{K_{3}\left(x_{10}\right) \rho\left(x_{10}\right)}} ; \\
\tau_{T} & =\tau_{1 T} \sqrt[4]{\frac{K_{3}\left(x_{10}\right) \rho\left(x_{10}\right)}{K_{3}\left(x_{1}\right) \rho\left(x_{1}\right)}} .
\end{aligned}
$$

From Eq. 60, Eq. 62 it follows that if $\sqrt[4]{\frac{K_{3}\left(x_{1}\right) \rho\left(x_{1}\right)}{K_{3}\left(x_{10}\right) \rho\left(x_{10}\right)}}<1$ then a wave profile takes place a compression. Therefore, the rate displacement wave has the compression and the shear stress wave has the decompression (Fig. 4).

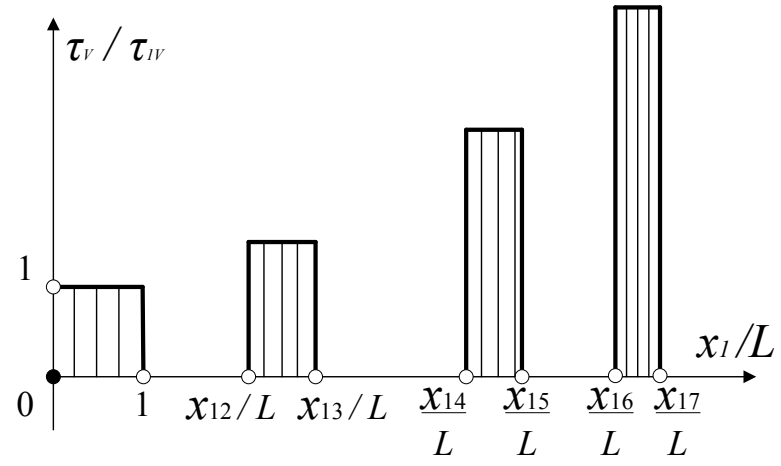

Figure 4. Dependency rate displacement $\tau_{\vartheta} / \tau_{1 \vartheta}$ on $x_{1} / L$ for shear wave of rectangular impulse.

Consider a propagation of shear wave which has 
triangular profile. In this case we can calculate analogous to rectangular impulse. The results of these calculations are presented schematically on Fig. 5

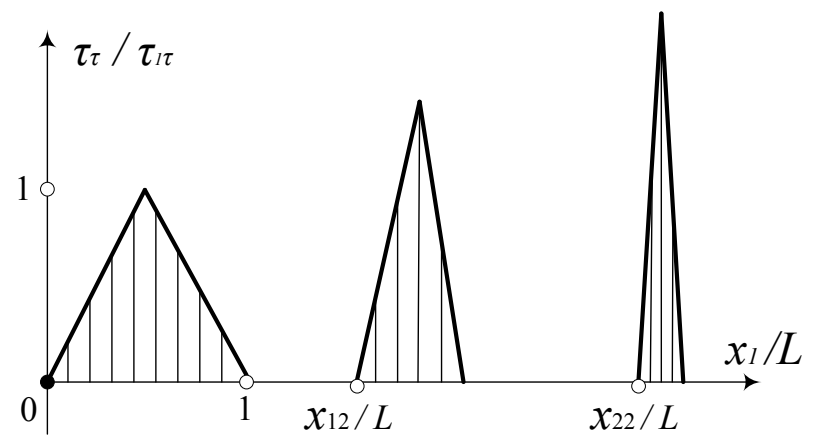

Figure 5. Dependency rate displacement $\tau_{\tau} / \tau_{1 \tau}$ on $x_{1} / L$ for shear wave of triangular impulse

The schematically results of propagation of shear wave which has triangular profile

\section{Oblique Wave Incidence on Boundary of Layer}

Consider the oblique wave incidence on the boundary $x_{1}=x_{10}$ from the half space $x_{1}<x_{10}$. In this case the rays will be curve lines which satisfy to the equations [17].

$$
\frac{d \bar{x}}{d s}=\bar{\tau}, \frac{d(n \bar{\tau})}{d s}=\operatorname{gradn}, \frac{d \psi}{d s}=n,
$$

where $\bar{\tau}$ is a tangential vector to ray trajectory, $n$ is a refraction coefficient, $s$ is a length along a ray.

For the systems of differential equations Eq. 61 we set boundary conditions on initial surface

$$
\begin{gathered}
\bar{x}=\bar{x}_{0}, \quad \bar{\tau}=\dot{\bar{x}}=\dot{\bar{x}}_{0}, \quad \psi=\psi_{0} \text { for } \quad S=S_{0} \\
\left(x_{1}=x_{10}\right) .
\end{gathered}
$$

If ray trajectory is found so surface (eiconal) $\psi$ is calculated along a ray accordingly do formula $\psi\left(\bar{x}_{1}\right)=\int_{M_{0}}^{M_{1}} \frac{d s}{C\left(\bar{x}_{1}\right)}=\int_{M_{0}}^{M_{1}} n\left(\bar{x}_{1}\right) d s \quad[7, \quad 17, \quad 18] . \quad$ Ray trajectories are orthogonal to surface $\psi\left(\bar{x}_{1}\right)=$ const .

In the formulas Eqs. 52, 53 a change of $d S$ along ray trajectories is found from Eq. 63. This dependence can't be found in analytical form for general case 3-D arbitrary function $n\left(x_{i}\right), i=1,2,3$.
Consider the Eq. 63 in plane $x_{1} O x_{2}$

$$
\frac{d(n \sin \theta)}{d S}=\frac{\partial n}{\partial x_{1}}=0 \text {. }
$$

The equation for ray trajectory has the form [17]

$$
\frac{d x_{2}}{d x_{1}}=\operatorname{tg} \theta=\frac{\sin \theta_{0}}{\sqrt{n^{2}-\sin ^{2} \theta_{0}}} .
$$

Set $L$ be $500 \mathrm{~m}$ then we have

$$
n^{2}=\sqrt{\frac{500}{500-x_{1}}} .
$$

where $n\left(x_{1}, x_{2}\right)=C_{0} / C_{S}\left(x_{1}, x_{2}\right)$ is a refraction coefficient.

The behavior of rays for different angles between an axis $x_{1}$ and rays is depicted on Fig. 6. How it follows from Eqs. $66,67 d n / d x_{1}>0$, then ray trajectories which have the angle $\theta_{0}<\frac{\pi}{2}$ become bent and drew near to the vertical. Therefore the wave front, which incidences on the free surface $x_{10}+L$, is parallel to plane $x_{1}=x_{10}+L$ and a refraction wave is plane, too.

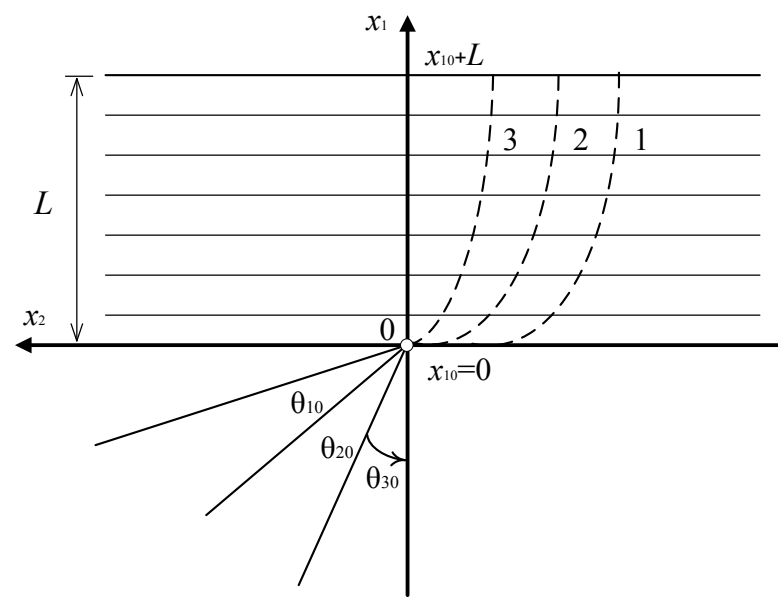

Figure 6. The trajectories of wave rays in stratified layer in vicinity of free surface of half-space

\section{The Reflection Wave which Propagates in the Direction $-x_{1}$ from the Free Surface $x_{1}=x_{10}+L$}

In this case solutions of the wave equations have the form of ray series 


$$
\begin{aligned}
& \vartheta_{2}\left(x_{1}, t\right)=T\left(x_{1}\right) \sum_{n=0}^{\infty} \vartheta_{2(+)}^{(n)} f_{n}^{(+)}(t+\psi)+R\left(x_{1}\right) \sum_{n=0}^{\infty} \vartheta_{2(-)}^{(n)} f_{n}^{(-)}(t-\psi), \\
& T_{12}\left(x_{1}, t\right)=T\left(x_{1}\right) \sum_{n=0}^{\infty} T_{12(+)}^{(n)} f_{n}^{(+)}(t+\psi)+R\left(x_{1}\right) \sum_{n=0}^{\infty} T_{12(-)}^{(n)} f_{n}^{(-)}(t-\psi) .
\end{aligned}
$$

The equations of a transfer we obtain if substitute Eqs. 68, 69 into Eqs. 26, 27.

$$
\begin{aligned}
& 2 K_{3} \psi^{\prime} \vartheta_{2(+)}^{(n)}+\left(K_{3} \psi^{\prime}\right) \vartheta_{2(+)}^{(n)}=K_{3} \vartheta_{2(+)}^{(n-1)^{\prime \prime}}+K_{3}^{\prime} \vartheta_{2(+)}^{(n-1)^{\prime}} \\
& 2 K_{3} \psi^{\prime} \vartheta_{2(-)}^{(n)}+\left(K_{3} \psi^{\prime}\right)^{\prime} \vartheta_{2(-)}^{(n)}=K_{3} \vartheta_{2(-)}^{(n-1)}{ }^{\prime \prime}+K_{3}^{\prime} \vartheta_{2(-)}^{(n-1)}{ }^{\prime} \\
& 2 K_{3} \psi^{\prime} T_{12(+)}^{(n)}+\left(K_{3} \psi^{\prime}\right) T_{12(+)}^{(n)}=K_{3} T_{12(+)}^{(n-1)}{ }^{\prime \prime}+K_{3}^{\prime} \vartheta_{2(+)}^{(n-1)}{ }^{\prime} \\
& 2 K_{3} \psi^{\prime} T_{12(-)}^{(n)}+\left(K_{3} \psi^{\prime}\right)^{\prime} T_{12(-)}^{(n)}=K_{3} T_{12(-)}^{(n-1)}{ }^{\prime \prime}+K_{3}^{\prime} \vartheta_{2(-)}^{(n-1)^{\prime}}
\end{aligned}
$$

where mark $(+)$ designates direct reflection wave and $(-)$ designates reverse (scattering) wave, $T\left(x_{1}\right), R\left(x_{1}\right)$ are coefficients of passage and reflection respectively.

The solutions of the Eqs. 70, 71 may be written in the form Eqs. 41, 44. We consider a principal part of ray series for $n=0$

$$
\begin{aligned}
& 2 K_{3} \psi^{\prime} v_{2(+)}^{(0)}+\left(K_{3} \psi^{\prime \prime}+K_{3}^{\prime} \psi^{\prime}\right) v_{2(+)}^{(0)}=0 \\
& 2 K_{3} \psi^{\prime}{\vartheta_{2(-)}^{(0)}}^{\prime}+\left(K_{3} \psi^{\prime \prime}+K_{3}^{\prime} \psi^{\prime}\right) \vartheta_{2(-)}^{(0)}=0 \\
& 2 K_{3} \psi^{\prime} T_{12(+)}^{(0)}+\left(K_{3} \psi^{\prime \prime}+K_{3}^{\prime} \psi^{\prime}\right) T_{12(+)}^{(0)}=0 \\
& 2 K_{3} \psi^{\prime} T_{12(-)}^{(0)}+\left(K_{3} \psi^{\prime \prime}+K_{3}^{\prime} \psi^{\prime}\right) T_{12(-)}^{(0)}=0 .
\end{aligned}
$$

We set the boundary conditions for the direct wave $(+)$

$$
v_{2(+)}^{(0)}\left(x_{10}+L\right)=v_{2}^{(0)}\left(x_{10}+L\right), \quad T_{12(+)}^{(0)}\left(x_{10}+L\right)=T_{12}^{(0)}\left(x_{10}+L\right) .
$$

The solutions of the Eqs. 72,74 with a registration Eq. 76 have the forms

$$
\begin{aligned}
& \vartheta_{2(+)}^{(0)}\left(x_{1}\right)=\vartheta_{2}^{(0)}\left(x_{10}+L\right) \sqrt[4]{\frac{K_{3}\left(x_{1}\right) \rho\left(x_{1}\right)}{K_{3}\left(x_{10}+L\right) \rho\left(x_{10}+L\right)}} \\
& T_{12(+)}^{(0)}\left(x_{1}\right)=T_{12}^{(0)}\left(x_{10}+L\right) \sqrt[4]{\frac{K_{3}\left(x_{10}+L\right) \rho\left(x_{10}+L\right)}{K_{3}\left(x_{1}\right) \rho\left(x_{1}\right)}}
\end{aligned}
$$

The refracted wave may be written with the help of the formulas $[7,19]$ in the form

$$
\begin{aligned}
& \vartheta_{2(-)}^{(0)}\left(x_{1}\right)=R\left(x_{1}\right) \vartheta_{2(+)}^{(0)}\left(x_{1}\right), \\
& T_{12(-)}^{(0)}\left(x_{1}\right)=R\left(x_{1}\right) T_{12(+)}\left(x_{1}\right),
\end{aligned}
$$

where $R\left(x_{1}\right)$ is a function which satisfies to Riccatti's equations $[18,19]$

$$
R^{\prime}=\frac{Q^{\prime}}{4 Q}\left(e^{2 i \psi}-R^{2} e^{-2 i \psi}\right), \quad Q\left(x_{1}\right)=C_{3}^{-2}\left(x_{1}\right) .
$$

Coefficients $T\left(\bar{x}_{1}\right), R\left(\bar{x}_{1}\right)$ allow taking into account an effect of a decreasing of an energy of direct wave and increasing of reflective wave energy. As it is known $[18,19]$ 


$$
R^{2}+T^{2}=1,
$$

thus if we know the solution of an Eq. 80 so it is possible to obtain a boundary condition for $\vartheta_{2(-)}^{(0)}, T_{12(-)}^{(0)}$ on the wave surface $\left(t+\psi\left(x_{1}\right)\right)$ in the formulas Eq. 79 .

The solutions for reflective waves have following expressions

$\vartheta_{2(-)}^{(0)}\left(x_{1}\right)=R\left(x_{1}\right) \vartheta_{2}^{(0)}\left(x_{10}+L\right) \sqrt[4]{\frac{K_{3}\left(x_{1}\right) \rho\left(x_{1}\right)}{K_{3}\left(x_{10}+L\right) \rho\left(x_{10}+L\right)}}$

$T_{12(-)}^{(0)}\left(x_{1}\right)=R\left(x_{1}\right) T_{12}^{(0)}\left(x_{10}+L\right) \sqrt[4]{\frac{K_{3}\left(x_{10}+L\right) \rho\left(x_{10}+L\right)}{K_{3}\left(x_{1}\right) \rho\left(x_{1}\right)}}$

Therefore, the Eqs. 77, 78, 80, 82, 83 gives us the solution of the problem about the propagation of reflection wave in the hypoplastic layer.

On Fig. 7 is depicted the dependency of $\vartheta_{2(+)}^{(0)}$ and $\vartheta_{2(-)}^{(0)}$ for $x_{1} / L=1(1), \quad x_{1} / L=0,9(2), \quad x_{1} / L=0,5(3)$, $x_{1} / L=0(4)$.

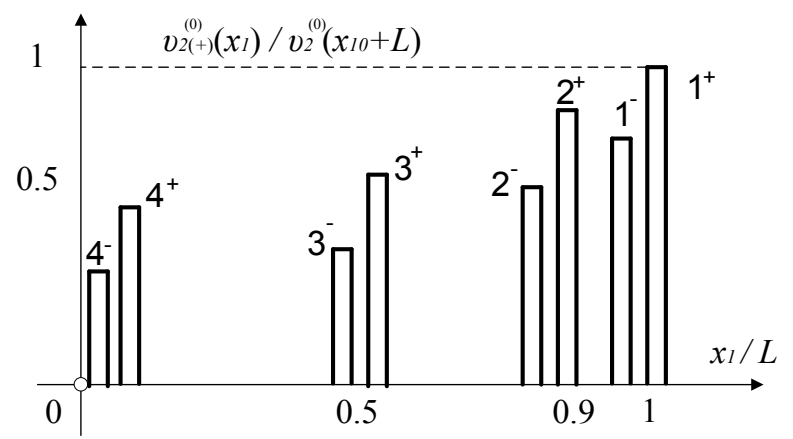

Figure 7. The dependency of $\vartheta_{2(+)}^{(0)}$ and $\vartheta_{2(-)}^{(0)}$ for $x_{1} / L=1(1)$, $x_{1} / L=0,9(2), x_{1} / L=0,5(3), x_{1} / L=0(4)$

On Fig. 8 is depicted the dependency of $T_{12(+)}^{(0)}, T_{12(-)}^{(0)}$ for same values

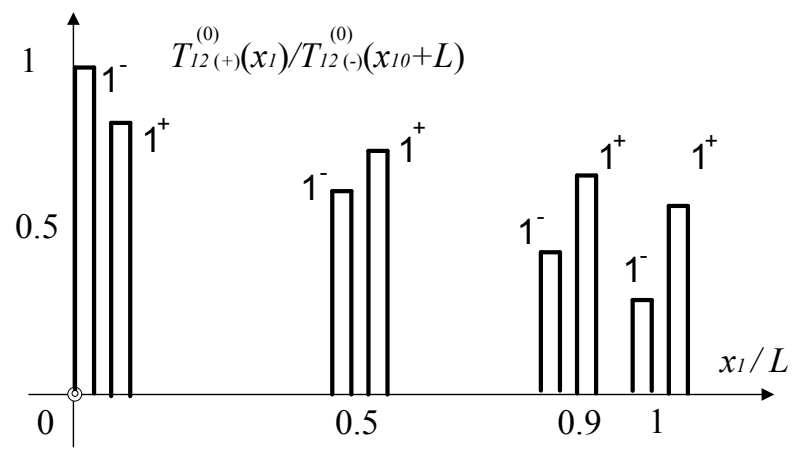

Figure 8. The dependency of $T_{12(+)}^{(0)}, T_{12(-)}^{(0)}$ for $x_{1} / L=1(1)$, $x_{1} / L=0,9(2), x_{1} / L=0,5(3), x_{1} / L=0(4)$

\section{Conclusions}

Shear waves arises in sources of earthquakes and propagate in direction of the earth surface. In the paper is described the process of shear wave which is arisen and propagates in stratified layer in free surface vicinity. A change of wave amplitude depends on wave resistance of effective medium which decreases in direction of free surface. In this case amplitudes of a displacement and a rate displacement increase but an amplitude of shear stress decreases. From energy law conversation, in the case a disturbance in the form of impulse function we obtain, that an impulse high increases for displacements and displacement rates but a width decreases. It is on contrary for a stress disturbance. An increasing of displacement and displacement rate is analogous to tsunami effect in case when a wave goes to a bank, however, a bend of a wave comb is absent. A wave, reflected from a free surface,

propagates in the direction $-x_{1}$ and is summed from a direct and a reflective wave. From the energy conservation law, it follows that an amplitude of a direct wave decreases and an amplitude of a reflective wave accumulates and increases in the direction of a free surface. A combined application of effective medium method and of ray method allows solving the problems of wave propagation in a stratified medium. A model of a hypoplastic medium is applied in the capacity of an effective medium. This approach is correct if thickness $l_{i}$ of each layer is more less then $L \quad\left(l_{i}<l, i=1,2, \ldots, n\right)$. 


\section{A List of Symbols}

$\vartheta(x, t)-$ velocity

$K$ - kinetic energy

$U$ - internal energy

$u(x, t)$ - specific internal energy of unit mass

$W$ - power of generalized mass forces $\mathrm{F}$

$F$ - mass forces

$Q$ - heat power

$t_{i j}-$ stress tensor

$V$ - volume of current pipe

$S$ - surface of current pipe

$t$ - time

$\bar{n}$ - unit normal vector

$\bar{P}$ - Umov's - Poyinting's vector

$L$ - thickness of layer

$l_{i}$ - thickness of i-layer

$f$-density of mass force vector

$T$ - tensor of effective stresses

$D_{i j}$ - tensor of rate

$\omega_{i j}-$ spin tensor

$e$ - pore quantity

$C_{S}$ - velocity of shear-wave

$n\left(x_{1}\right)$ - refraction coefficient

$R\left(x_{1}\right)$ - coefficient of reflection

$T\left(x_{1}\right)$ - coefficient of transfer

$\bar{\tau}$ - unit tangential vector

$\rho$ - coefficient of reflection

$\psi$ - eiconal

$\theta$ - angle

$\dot{T}$ - material derivative time

$x_{i}-$ Cartesian coordinate

$\stackrel{\circ}{T}$ - Jaunman's derivative time
349, (1978).

[4] Delph, T.J., Herrmann, G. and Kaul, R.K. Harmonic Wave Propagation in a Periodically Layered, Infinite Elastic Body: Plane Strain, Numerical Results, New York: American Society of Mechanical Engineers, Journal of Applied Mechanics, 47(3), (1980).

[5] Brekhovskikh, L. M. Waves in Layered Media, Academic Press, (1980).

[6] Rossikhin, Yu. A., Shitikova, M.V. Ray method for solving dynamic problems connected with the propagation of wave surfaces of strong and weak discontinuities, Appl. Mech. Reviews, 48(1), 1-39, (1995).

[7] Chigarev, A.V. Stochastic and regular dynamics of heterogeneous environments [Russian translation], Minsk: UE “Tehnoprint", (2000).

[8] Osinov, V.A., Gudehus, G. Dynamics of Hypoplastic Materials: Theory and Numerical Implementation, In: Deformation and Failure of Granular an Porous Continua, N. Kirchler, K. Hutter eds., Lecture Notes in Applied Mechanics, Springer, Berlin, (2003).

[9] Osinov, V., Gudehus, G. Plane shear waves and loss of stability in a saturated granular body. Mechanics of Cohesive-fritional Materials, 1(1), 25-44, (1996).

[10] Kolarov, D., Baltov, A. and Boncheva, N. Mechanics of Plastic Media [Russian translation], Mir Publ., Moscow, (1979).

[11] Sedov, L.I. Mechanics of continuous media [Russian translation], Moscow: Science, 1, (1979).

[12] Brown, Dm. N. Physics of strong motions excited by earthquakes. Seismic Risk and Engineering Decisions, Elsevier, Amsterdam - Oxford - New York, 129-161, (1976).

[13] Kanamori, H. (Ed.) Earthquake Seismology: Treatise on Geophysics, Elsevier, (2009).

[14] Barkan, D. D. Dynamics of bases and foundations, New York, McGraw Hill, (1962).

[15] Cravtsov, Jn. A., Orlov, Jn. I. Geometrical optics of inhomogeneous media [Russian translation], M. Science, (1980).

[16] Vinogradova, M. B.; Rudenko, O. V.; Sukhorukov, A. P. Theory of waves: textbook for physics qualifications in higher education [Russian translation], Moscow: Nauka, (1990).

[17] Babkin, G. I., Klyatskin, V. I. Invariant imbedding method for wave problems, Wave Motion, 4(2), 195-207, 1982.

[18] Klyatskin, V I, The Imbedding Method in Wave Propagation Theory [Russian translation], Moscow: Nauka, (1986).

[19] Klyatskin, V. I. Dynamics of Stochastic Systems, Elsevier Science, (2005). 